\title{
REDUÇÃO NO TEMPO DE SETUP NA MÁQUINA DE TELA DE COLUNA*
}

Raul Alcantara Batista ${ }^{1}$ Moema Cianelli Cezar Avelar ${ }^{2}$

Diogo Barcellos Moraes ${ }^{3}$

\section{Resumo}

O presente trabalho tem como premissa abordar estudos baseados na metodologia Troca Rápida de Ferramentas (TRF ou em inglês, SMED - Single-Minute Exchange of Die), e realizar um estudo de caso na empresa Votorantim Siderurgia. O objetivo principal é a redução do tempo de setup da máquina de Tela de Coluna, a fim de minimizar o desperdício de tempo e maximizar a produtividade. Além disso, obteve ganhos efetivos no layout de ferramentas, programa $5 \mathrm{~S}$, tempo de atividades, entre outros.

Palavras-chave: Setup; SMED.

\begin{abstract}
REDUCTION IN SETUP TIME IN A COLUMN MESH MACHINE
Abstract

The present work has as premise to approach studies based on the methodology Troca Rápida de Ferramentas (TRF or SMED - Single-Minute Exchange of Die) and carry out a case study at Votorantim Siderurgia company. The main goal is the Setup time reduction in the column mesh machine in order to minimize wasted time and maximize productivity. In addition, effective gains were obtained in tool layout, 5S program, time of activities, among others.
\end{abstract}

Keywords: Setup; SMED.

1 Engenheiro Mecânico, Gerente de Acabamento Votorantim Siderurgia, Resende, Rio de Janeiro, Brasil.

2 Cursando Engenharia de Produção, Estagiária na Gerência de Acabamento, Votorantim Siderurgia, Resende, Rio de Janeiro, Brasil.

3 Técnico Metalúrgico, Técnico Especialista, Votorantim Siderurgia, Resende, Rio de Janeiro, Brasil. 


\section{INTRODUÇÃO}

Este trabalho irá abordar a otimização do tempo de setup numa máquina que fabrica Telas de Coluna, a fim de implantar melhorias que reduzissem os procedimentos envolvidos nas atividades dos funcionários.

De acordo com um levantamento realizado no ano de 2015, a duração média de setup era equivalente a $4,9 \mathrm{~h}$, sendo que sua frequência era de 30 setups/mês. Então, através da análise de disponibilidade, foi visto a possibilidade de melhoria, pois, não existia uma padronização das atividades. A célula apresentava desorganização, o layout não era adequado, entre outras oportunidades, criando assim um ambiente propício a muitas perdas, sobrecarga, desperdício de mão de obra e tempo nas atividades.

\section{MATERIAIS E MÉTODOS}

O projeto irá abordar sobre uma pesquisa aplicada, pois a principal intenção é resolver problemas concretos dentro da empresa, ou seja, na célula da Tela de Coluna. Esse problema será tratado com o desenvolvimento de estudo de caso e uma pesquisa de campo, abrangendo também uma pesquisa quantitativa e qualitativa para levantar dados da otimização da redução do tempo de setup.

Para o levantamento de dados, foi realizado uma pesquisa de campo, para efetuar a coleta.

A metodologia utilizada foi a Troca Rápida de Ferramentas (TRF ou em inglês, SMED - Single-Minute Exchange of Die), é um método utilizado para analisar a produção em uma linha de montagem industrial, com a intenção de reduzir o tempo gasto pelos ajustes e trocas de peças entre diferentes produções. Foi desenvolvida pelo japonês Shigeo Shingo consultor da Toyota. O princípio surgiu a partir da década de 1950. [1]

De acordo metodologia TRF (Troca rápida de Ferramentas), existem estágios que devem ser seguidos para a implantação [2]. São elas:

- Estágio preliminar: Estudar com detalhes as reais condições da linha de produção. Esses estudos podem ser auxiliados através de cronômetros e filmagens. 
- Estágio 1: É a separação entre os setups internos e externos. As técnicas devem garantir que a maioria das tarefas sejam realizadas como setup externo.

Conceitualmente os setups são classificados da seguinte forma:

1. Setup Interno: procedimentos que podem ser executados apenas quando a máquina estiver parada.

2. Setup Externo: procedimentos que podem ser executados enquanto a máquina está em operação.

- Estágio 2: É a conversão do setup interno em externo. Nesse estágio deve-se encontrar meios de converter as atividades internas, a padronização de acessórios, ferramentas ou de funções.

- Estágio 3: É a racionalização das operações de setup. Necessita-se de uma análise de todas as operações para reduzir determinas atividades, seja ela interna ou externa. As técnicas desenvolvidas para essa fase são a melhoria radical nas operações de setup externo realizadas ao redor da máquina, como estantes, ferramentas e acessórios, que embora não reduzam o tempo de setup final, evita desgaste físico desnecessário do operador ao realizar sua tarefa.

\section{RESULTADOS E DISCUSSÃO}

A pesquisa foi realizada na empresa Votorantim Siderurgia localizada em Resende RJ. Para comprovar que o tempo de setup influencia diretamente na produção, foi analisado um gráfico de Pareto, onde se confirma a necessidade de melhoria em relação ao tempo do setup, pois se destaca como principais motivos de perda de produção. 


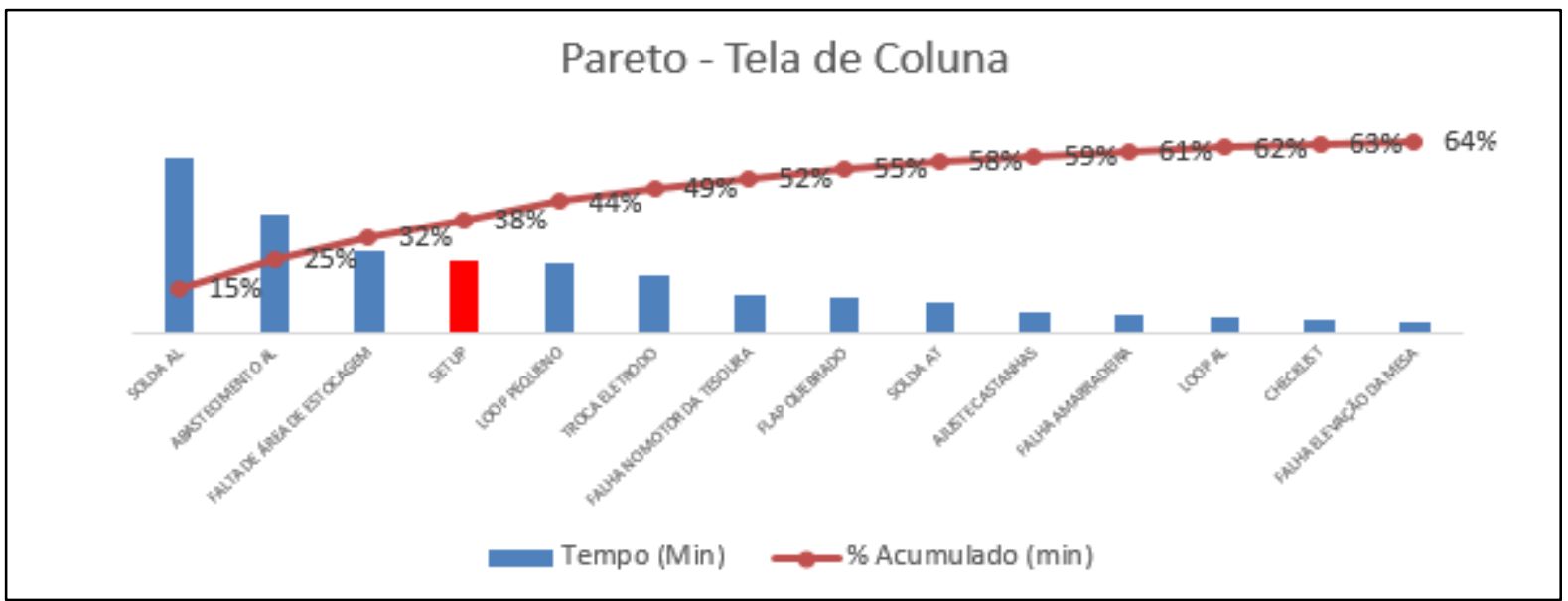

Figura 1. Pareto Tela MEP - Principais de Perda de Produção Fonte: Criada pelo autor

No gráfico de Pareto, verifica-se os principais motivos de perda de produção que influenciam no índice de utilização. Os três mais relevantes são: solda no arame longitudinal ( $\mathrm{AL}$ ), abastecimento do arame longitudinal e falta de estocagem. Entretanto, são itens que não entram no escopo do projeto, pois precisam de um alto investimento (CAPEX) para que os problemas sejam solucionados. Por conta disso, o foco foi voltado ao tempo de setup.

Para iniciar as atividades do projeto, foi realizada uma estimativa, com os objetivos e o ganho potencial. Segue os dados referentes às informações:

Tabela 1. Tabela Resumo de Ganhos Potenciais

\begin{tabular}{|c|c|c|c|}
\hline Objetivo & Atual & Meta & Ganho Potencial \\
\hline $\begin{array}{c}\text { Reduzir o tempo } \\
\text { de Setup }\end{array}$ & 4,9 horas & 2,5 horas & $\begin{array}{c}\text { Ganho de } 0,70 \% \\
\text { de utilização }\end{array}$ \\
\hline
\end{tabular}

Fonte: Criada pelo autor

De acordo com a metodologia, seguem os estágios:

- Estágio Preliminar: Foi verificado que a duração da atividade era de 4,9h. Essa duração está relacionada principalmente à falta de organização e padronização. Em relação à quantidade de funcionários que participaram da atividade, foram escalados dois operadores. Abaixo, é mostrada uma tabela com o acompanhamento realizado.

Tabela 2. Condição Atual

\begin{tabular}{|l|c|c|c|c|}
\hline \multicolumn{5}{|c|}{ Condição Atual } \\
\hline \multicolumn{1}{|c|}{ Setup } & Início & Fim & Hora & Medição real \\
\hline Tracionador intermediário & $09: 29$ & $10: 16$ & $00: 47$ & 47 \\
\hline Mesa endireitadora & $08: 04$ & $08: 10$ & $00: 06$ & 6 \\
\hline
\end{tabular}




\begin{tabular}{|l|c|c|c|c|}
\hline Mesa de espaçamento & $08: 14$ & $09: 29$ & $01: 15$ & 75 \\
\hline Cabeçote de soldagem & $10: 00$ & $12: 41$ & $02: 41$ & 161 \\
\hline Mesa de painéis & $12: 47$ & $12: 52$ & $00: 05$ & 5 \\
\hline \multicolumn{3}{|c|}{ TOTAL (Min) } & 294 \\
\hline TOTAL (H) & 4,9 \\
\hline
\end{tabular}

Fonte: Tabela Criada pelo autor

Além do tempo, foi verificado o deslocamento que os operadores percorriam para que a atividade fosse finalizada. Foi calculado que ao total de um ano eles iriam transitar certa de $116 \mathrm{~km}$. Segue abaixo o esquema conhecido como "Gráfico de Spaghetti" realizado durante o acompanhamento.

\section{Condição Atual}

\section{Spaghetti Deslocamento para Setup}
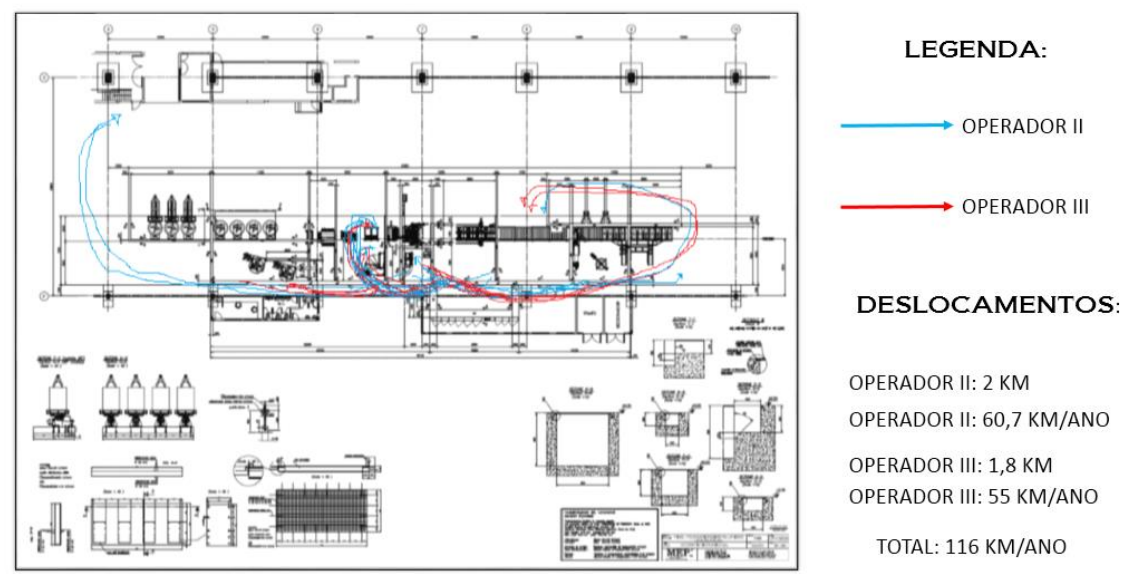

Figura 2. Spagheti Deslocamento para Setup Fonte: Gráfico criado pelo autor

- Estágios 1 e 2: Para essas etapas, durante o acompanhamento foi verificada a situação das atividades que eram internas e externas e sugerido uma proposta de conversão das atividades. A tabela 3 apresenta as informações: 
Tabela 3. Levantamento de atividades internas e externas com proposta

\begin{tabular}{|c|c|c|c|c|c|c|}
\hline \multirow[t]{2}{*}{ Equipamento } & \multicolumn{2}{|r|}{ Atividades } & \multirow{2}{*}{$\frac{\text { Tempo }}{\text { (min) }}$} & \multirow{2}{*}{$\begin{array}{l}\text { Antes } \\
\text { do } \\
\text { SMED } \\
\text { Interno }\end{array}$} & \multicolumn{2}{|c|}{ Proposta } \\
\hline & $\mathrm{N}^{\circ}$ & Descrição & & & Interno & Externo \\
\hline \multirow{5}{*}{$\begin{array}{l}\text { Mesa de } \\
\text { Espaçamento }\end{array}$} & 1 & Retirada da mesa & 5 & $\mathrm{X}$ & $\mathrm{X}$ & \\
\hline & 2 & Ajuste da mesa & 38 & $\bar{X}$ & $\mathrm{X}$ & \\
\hline & 3 & Retirada de amostra & 5 & $\mathrm{X}$ & & $\mathrm{X}$ \\
\hline & 4 & Fixação da mesa & 15 & $X$ & $X$ & \\
\hline & 5 & $\begin{array}{l}\text { Esperando op1 buscar } \\
\text { porca }\end{array}$ & 12 & $X$ & & $X$ \\
\hline \multirow{7}{*}{$\begin{array}{l}\text { Tracionador } \\
\text { Intermediário }\end{array}$} & 6 & $\begin{array}{l}\text { Preparando } \\
\text { ferramentas }\end{array}$ & 4 & $\mathrm{X}$ & & $X$ \\
\hline & 7 & $\begin{array}{lr}\text { Retirar material do } \\
\text { cabeçote } & \text { e } \\
\text { tracionador } & \\
\end{array}$ & 6 & $\mathrm{X}$ & $\mathrm{X}$ & \\
\hline & 8 & $\begin{array}{l}\text { Soltar parafusos de } \\
\text { fixação das placas do } \\
\text { tracionador }\end{array}$ & 3 & $X$ & $\mathrm{X}$ & \\
\hline & 9 & $\begin{array}{l}\text { Retirada do tubo do } \\
\text { arame transversal }\end{array}$ & 2 & $X$ & $X$ & \\
\hline & 10 & $\begin{array}{l}\text { Preparação da tela no } \\
\text { painel operacional }\end{array}$ & 3 & $X$ & $X$ & \\
\hline & 11 & $\begin{array}{l}\text { Ajuste tracionador } \\
\text { intermediário }\end{array}$ & 20 & $X$ & $X$ & \\
\hline & 12 & $\begin{array}{l}\text { Retirada das guias de } \\
\text { saídla do } A L\end{array}$ & 9 & $X$ & & $X$ \\
\hline \multirow{5}{*}{$\begin{array}{l}\text { Cabeçote de } \\
\text { Soldagem }\end{array}$} & 13 & Ajuste flap's e feeders & 17 & $X$ & $\mathrm{X}$ & \\
\hline & 14 & Alinhamento cilindros & 75 & $\bar{X}$ & $\mathrm{X}$ & \\
\hline & 15 & Vazamento de ar & 11 & $\mathrm{X}$ & & $X$ \\
\hline & 16 & $\begin{array}{l}\text { Esperando op1 buscar } \\
\text { parafuso do cilindro }\end{array}$ & 3 & $X$ & $X$ & \\
\hline & 17 & Arrumar ferrametas & 3 & $\bar{x}$ & & $\bar{X}$ \\
\hline
\end{tabular}




\begin{tabular}{|c|c|l|c|c|c|c|}
18 & Troca de eletrodo & 4 & $\mathrm{X}$ & $\mathrm{X}$ \\
\cline { 2 - 6 } & 19 & Passagem de material & 6 & $\mathrm{X}$ & $\mathrm{X}$ & \\
\hline 20 & $\begin{array}{l}\text { Embolo arame } \\
\text { transversal da malha }\end{array}$ & 5 & $\mathrm{X}$ & $\mathrm{X}$ & \\
\hline 21 & $\begin{array}{l}\text { Parafuso da } \\
\text { quebrado }\end{array}$ & $\mathrm{X}$ & & $\mathrm{X}$ \\
\hline
\end{tabular}

Fonte: Criada pelo autor

Com essa sugestão as atividades sofreram alterações em relação à distribuição de execução interna e externa. As tabelas 4, 5 e 6 mostram os ganhos obtidos em relação à conversão de atividades.

Tabela 4. Ganho em atividades externas na Mesa de Espaçamento

\begin{tabular}{|l|c|c|c|}
\hline $\begin{array}{c}\text { Mesa de } \\
\text { espaçamento }\end{array}$ & $\begin{array}{c}\text { Tempo } \\
\text { Condição Atual } \\
\text { (min) }\end{array}$ & $\begin{array}{c}\text { Proposta } \\
\text { (Min) }\end{array}$ & $\begin{array}{c}\text { Ganho em atividades } \\
\text { externas }\end{array}$ \\
\hline Interno & 75 & 58 & $23 \%$ \\
\hline Externo & 0 & 17 & 23 \\
\hline \multicolumn{2}{|c|}{ Fonte: Criada pelo autor } \\
\hline
\end{tabular}

Tabela 5. Ganho em atividades externas no Tracionador Intermediário

\begin{tabular}{|l|c|c|c|}
\hline $\begin{array}{c}\text { Tracionador } \\
\text { intermediário }\end{array}$ & $\begin{array}{c}\text { Tempo } \\
\text { Condição Atual } \\
\text { (Min) }\end{array}$ & $\begin{array}{c}\text { Proposta } \\
\text { (Min) }\end{array}$ & $\begin{array}{c}\text { Ganho em atividades } \\
\text { externas }\end{array}$ \\
\hline Interno & 47 & 34 & \\
\hline Externo & 0 & 13 & $28 \%$ \\
\hline
\end{tabular}

Fonte: Criada pelo autor

Tabela 6. Ganho em atividades externas no Cabeçote de Soldagem

\begin{tabular}{|l|c|c|c|}
\hline $\begin{array}{c}\text { Cabeçote de } \\
\text { soldagem }\end{array}$ & $\begin{array}{c}\text { Tempo } \\
\begin{array}{c}\text { Condição Atual } \\
\text { (Min) }\end{array}\end{array}$ & $\begin{array}{c}\text { Proposta } \\
\text { (Min) }\end{array}$ & $\begin{array}{c}\text { Ganho em atividades } \\
\text { externas }\end{array}$ \\
\hline Interno & 161 & 106 & \multirow{2}{*}{$34 \%$} \\
\hline Externo & 0 & 55 & \\
\cline { 1 - 2 }
\end{tabular}

- Estágio 3: A fim de racionalizar as operações de setup, foram implementadas ações de melhoria, como check-list, 5S, sequência de atividades dos funcionários, entre outras. A tabela abaixo descreve as ações:

\section{Ações de Melhoria}

$1 \quad$ Definir instruções de trabalho para setup geral

2 Criar check list de setup 


\begin{tabular}{|l|l|}
\hline $\mathbf{3}$ & $\begin{array}{l}\text { Confeccionar bancada próxima ao equipamento para ajuste de } \\
\text { espaçamento }\end{array}$ \\
\hline $\mathbf{4}$ & Desenvolver gabarito para ajuste de espaçamento do fio longitudinal \\
\hline $\mathbf{5}$ & Definir a função dos operadores II/III durante o setup \\
\hline $\mathbf{6}$ & Disponibilizar o carrinho de ferramentas próximo ao setup \\
\hline $\mathbf{7}$ & Definir ferramentas de uso no setup \\
\hline $\mathbf{1 0}$ & Definir guia com material correto do AL \\
\hline $\mathbf{1 2}$ & Definir parafuso para fixação das malhas condutoras \\
\hline $\mathbf{1 3}$ & Padronizar parafusos de feeders e flap's \\
\hline $\mathbf{1 4}$ & Realizar kanban nos parafusos de: cilindros, feeder e flap's \\
\hline $\mathbf{1 5}$ & Desenvolver mangueiras de ar comprimido flexíveis \\
\hline $\mathbf{1 6}$ & Posicionamento do cilindro antes de fixar o barramento \\
\hline $\mathbf{1 7}$ & Criar gabaritos para cada espaçamento de tela \\
\hline
\end{tabular}

Tabela 7. Ações de Melhoria

\section{Fonte: Tabela criada pelo autor}

Foram desenvolvidas ações de melhoria que auxiliariam a redução do tempo. A figura 3 apresenta um padrão segue proposto de atividades que cada funcionário deve seguir ao iniciar o setup. 


\begin{tabular}{|c|c|c|c|}
\hline \multicolumn{4}{|c|}{ SEQUENCIA PRDPOSTA } \\
\hline & Atividades & Recursos & $\begin{array}{l}\text { Preposta Tempo ( } \\
\text { min) }\end{array}$ \\
\hline 1 & $\begin{array}{l}\text { Separar no carrinho de ferramentas somente as chaves que } \\
\text { serio utlizades durante o cambie }\end{array}$ & OP & 0 \\
\hline 2 & Separar o gabarto correto para o ajuste de espaçamento & OPI & 0 \\
\hline 3 & Abrir o cabeçote de soldagem & OP & 1 \\
\hline 4 & Retirar material do cabeçote e da mesa de espaçamento & OPle Tesoura & 3 \\
\hline 5 & Retirar mesa de espaçamento & OPI/ 2 chave combinadas $24 \mathrm{~mm}$ & 5 \\
\hline 6 & Disponbilizar gabarto correto próximo a mess de espaçamento & OPI & 1 \\
\hline 7 & Retirada do tubo do arame transversal & OP II / chave alen $6 \mathrm{~mm}$ & 6 \\
\hline 8 & Mudança de tela no painel operacional & OP & 2 \\
\hline 9 & Sottar parafusos de foxação das placas do conjunto tracionador & OP I/ chave catraca e cachimbo $22 \mathrm{~mm}$ & 4 \\
\hline 10 & Soltar parafusos dos flap's e feeder's & OP I/ chave combinada $14 \mathrm{~mm}$ & 7 \\
\hline 11 & Sotar paratusos dos clindros de soldagem e mahas & OP I/ chave alen $8 \mathrm{~mm}$ & s \\
\hline 12 & Ajustar mesa de espacamento & $\begin{array}{l}\text { OP II/gabarto de espaçamento / chave } \\
\text { combinada } 17 \mathrm{~mm} / \text { chave alen } 8 \mathrm{~mm}\end{array}$ & 10 \\
\hline 13 & Focação da mesa & OP 1/2 chave combinadas $24 \mathrm{~mm}$ & 15 \\
\hline 14 & Ajuste do conjunto tracionador & OP I & 10 \\
\hline 15 & Apertar parafusos de fxaçilio das placas & $\begin{array}{l}\text { OP I/OP I / chave catraca e cachimbo } \\
222 \mathrm{~mm}\end{array}$ & 5 \\
\hline 16 & Ajuste e aperto dos parafusos dos flap's & OP E/ Chave combinada $14 \mathrm{~mm}$ & 5 \\
\hline 17 & Ajuste e aperto dos parafuses dos feeder's & OP E/ Chave combinada 14 mm & 5 \\
\hline 18 & Alnhamento e aperto dos parafusos dos cilindros & $\begin{array}{l}\text { OP I / gabarto de espaçamento / chave } \\
\text { alen } 8 \mathrm{~mm}\end{array}$ & 30 \\
\hline 19 & Passagem de material & OPI & 10 \\
\hline 20 & Resizar o zeramento & 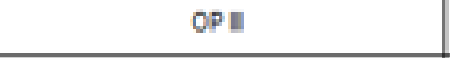 & 2 \\
\hline 21 & Testar a soldagem e mediçăo đimensionsl & OP $\mathbf{E}$ & 3 \\
\hline 22 & Ligar a máquina & OP $\mathbf{E}$ & 1 \\
\hline 23 & Alinhar batentes posicionadores & $O P=/ O P I$ & 5 \\
\hline 24 & Resalzar teste de dobra & $O P=/ O P I$ & 5 \\
\hline \multirow[t]{2}{*}{25} & Separar produção não conforme e descartar & OPI & 3 \\
\hline & \multicolumn{2}{|l|}{ Total } & $2 \mathrm{~h} 23 \min$ \\
\hline
\end{tabular}

Figura 3. Sequência Proposta de Setup

Fonte: Criada pelo autor

A tabela 8 apresenta um check list que o funcionário deverá realizar com 4 horas de antecedência, para facilitar a organização dos itens e não desperdiçar tempo durante o setup.

\section{Check list de Setup}

horas antes do início do Setup) 


\begin{tabular}{|c|c|c|c|}
\hline \multicolumn{4}{|c|}{ Produto: } \\
\hline \multicolumn{4}{|c|}{ Responsável: } \\
\hline $\mathrm{Da}$ & $1 /$ & & \\
\hline \multirow{2}{*}{\multicolumn{2}{|c|}{ SETUP - GERAL }} & \multicolumn{2}{|c|}{ CHECAGEM } \\
\hline & & Ok & Não OK \\
\hline 1 & Chave combinadas $24,22,19,17$ e $14 \mathrm{~mm}$ & & \\
\hline 2 & Chave catraca e cachimbo 24,22 e $19 \mathrm{~mm}$ & & \\
\hline 3 & Chaves allen 8 e 6 mm & & \\
\hline 4 & Parafusos de ajuste dos feeders & & \\
\hline 5 & Parafusos de ajuste dos flaps & & \\
\hline 6 & Parafusos das malhas condutoras & & \\
\hline 7 & Parafusos de fixação do eletrodo & & \\
\hline 8 & Parafusos de ajuste da placa tracionadora & & \\
\hline 10 & Porca de fixação da mesa & & \\
\hline 11 & Barramento das malhas condutoras & & \\
\hline 12 & Batentes da mesa empenado & & \\
\hline 13 & Parafusos da mesa de endireitamento & & \\
\hline 14 & Parafusos de fixação do cilindro de soldagem & & \\
\hline 15 & Rolamento dos rolos endireitadores & & \\
\hline
\end{tabular}

Tabela 8. Check List de Setup (4 horas antes do início do Setup)

Fonte: Tabela criada pelo autor

Foram identificadas oportunidades relacionadas ao $5 \mathrm{~S}$ da área, pois as ferramentas não ficavam organizadas e existia muita sujeira na área. Com a melhoria, os itens ficaram organizados, identificados e limpos. A figura 4 apresenta a modificação.

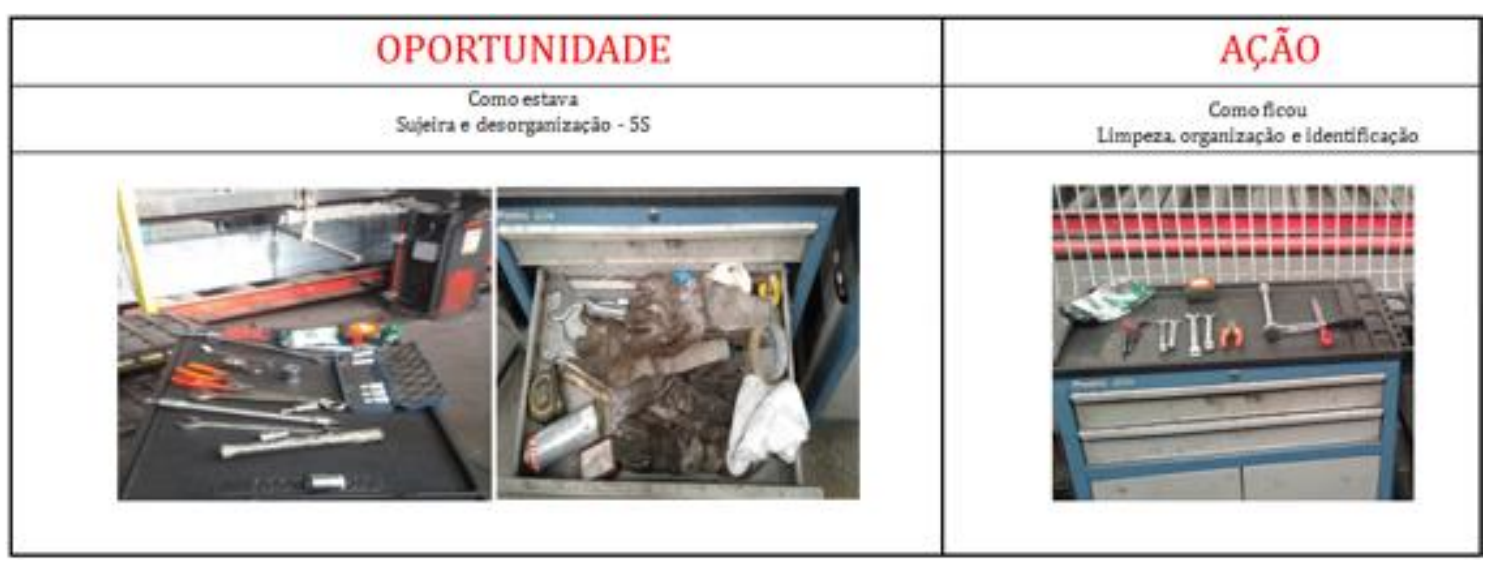

Figura 4. Oportunidade de Ações de 5S Fonte: Criada pelo autor

Depois que as ações foram implantadas, foi amostrado um novo setup, percebendo-se resultados positivos. A figura 5 apresenta um novo gráfico de Spaghetti, onde nitidamente o deslocamento dos operadores diminuiu com uma nova distância de $52 \mathrm{~km} / \mathrm{ano}$. 


\section{Condição Posterior}
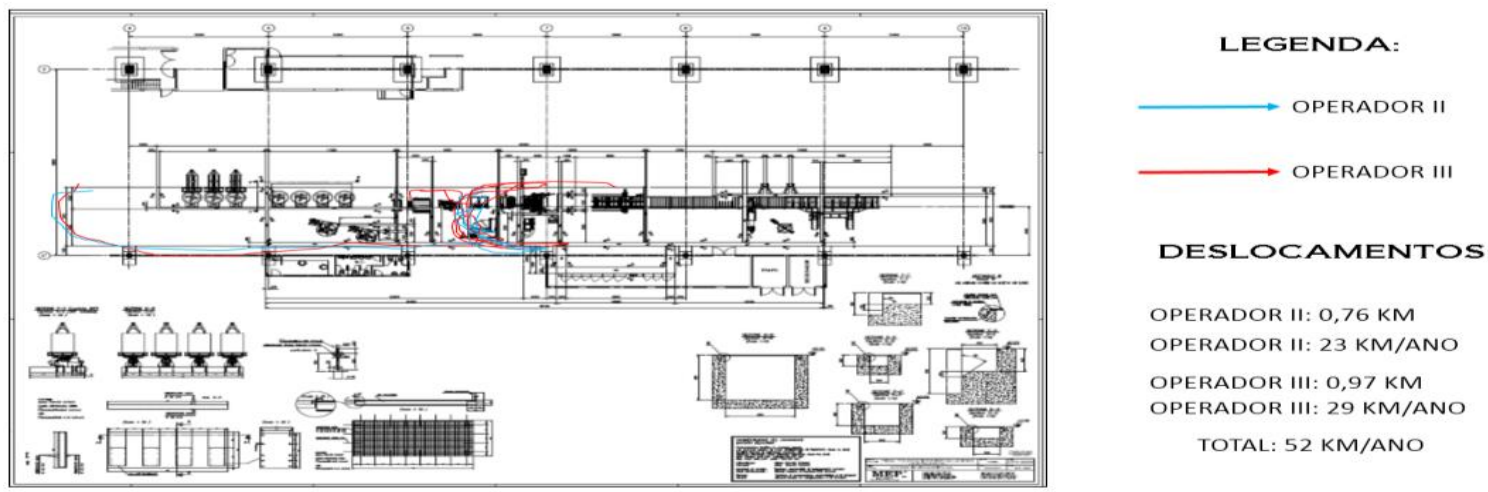

Figura 5. Spagheti Deslocamento para Setup Condição Posterior Fonte: Criada pelo autor

Por fim, a tabela 9 apresenta os ganhos obtidos com a execução da ferramenta SMED:

Tabela 9. Resultados

\begin{tabular}{|c|c|c|c|c|}
\hline Objetivo & Atual & Meta & Redução & Ganho \\
\hline Reduzir o tempo de Setup & 4,9 horas & $\begin{array}{c}2,23 \\
\text { horas }\end{array}$ & 2,03 & $\begin{array}{c}\text { Redução de } \\
58,60 \% \text { no tempo } \\
\text { de setup }\end{array}$ \\
\hline Deslocamento do operador & $116 \mathrm{~km} / \mathrm{ano}$ & - & $52 \mathrm{~km} / \mathrm{ano}$ & $\begin{array}{c}\text { Redução de } \\
55,17 \% \text { no } \\
\text { deslocamento }\end{array}$ \\
\hline
\end{tabular}

Foi analisado com o trabalho que o novo valor do tempo de setup foi alterado para 2,03h, ou seja, uma redução de $2,87 \mathrm{~h}$ por setup. Ao ano existe a possibilidade de incremento de 310 ton na produção.

\section{CONCLUSÃo}

A empresa comprovou a eficácia da aplicação do método Troca Rápida de Ferramenta (SMED), o qual gerou ganhos efetivos, pois além da redução do tempo de troca, o layout de localização das ferramentas foi alterado, assim como o $5 \mathrm{~S}$ sofreu modificações, os operadores não necessitam transitar sem necessidade durante a troca, assim reduzindo cansaço. Além disso, como o tempo dessas atividades reduziram, a produção sofreu um aumento significativo durante 0 ano. Como a aplicação da ferramenta é bem abrangente, a mesma pode ser implantada em diversos setores da empresa, gerando novos lucros. 


\section{Agradecimentos}

Agradecimento a todos os funcionários que se empenharam e dedicaram para que o projeto tivesse um ganho efetivo. São eles:

- Raul Alcantara Batista - Facilitador de Melhoria

- Carlos Diego - Facilitador de Rotina

- Leandro Pereira Leite - Técnico Especialista

- Diogo Barcellos Moraes - Técnico Especialista

- Moema Cianelli Cezar Avelar - Estagiária

- Thiago Von Rondon Duarte - Operador III

- André Macedo da Rocha - Operador III

- Jason Campos Gama - Operador III

- Rickson Silva Mendes - Operador II

- Ranon dos Santos Gonçalves - Operador II

- Edgar Alberto Rodrigues de Amorim - Operador II

- Baltazar Augusto Alves - Operador II

- Luiz Paula Moreira de Souza - Operador II

\section{REFERÊNCIAS}

1 Sugai M, Mclntosh R, Novaski O. Metodologia de Shigeo Shingo (SMED): análise crítica e estudo de caso. 2007 ; 14. 323-336 ; [acesso em 13 jun.2017] ; disponíevel em:http://jetaconsul.dominiotemporario.com/doc/SMED_Analise_Critica_e_Estudo_de_ Caso.pdf

2 Monteiro A, Menezes J O, M.Sc. Troca rápida de ferramentas aplicada a uma indústria siderúrgica do Rio de Janeiro: um estudo de caso. 2007; [acesso em 13 jun.2017]; diposnívelem:https://www.researchgate.net/publication/280489982_TROCA_RAPIDA DE_FERRAMENTAS_APLICADA_A_UMA_INDUSTRIA_SIDERURGICA_DO_RIO_DE JĀNEIRO_UM_ESTŪDO_DE_CĀSO 